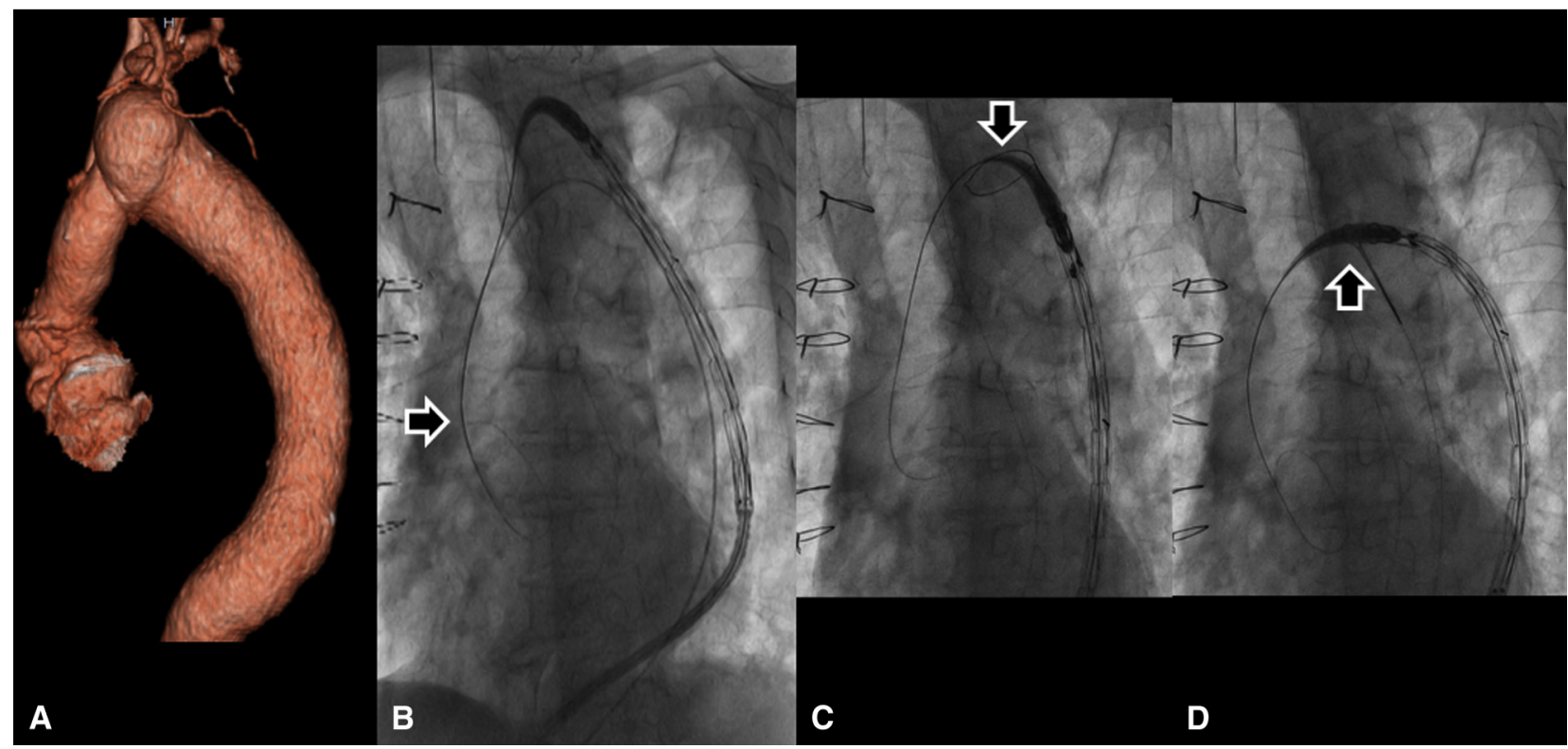

FIGURE 1. Lasso technique used in an 88-year-old man with a history of total arch replacement with a 24-mm multibranched polyester graft at the age of 70 years, abdominal aortic aneurysm replacement at 71 years, and replacement of the proximal ascending aorta with a $24-\mathrm{mm}$ polyester graft at 83 years. The aneurysm at the distal anastomosis of the arch graft developed at the top of the arch angulation. A, Left lateral projection of 3-dimensional computed tomography. B, C, and D, Fluoroscopic images during the lasso technique procedure. Arrows indicate snare.

\section{References}

1. Malkawi AH, Hinchliffe RJ, Yates M, Holt PJ, Loftus IM, Thompson MM. Morphology of aortic arch pathology: implications for endovascular repair. J Endovasc Ther. 2010;17:474-9.

2. Joseph G, Stephen E, Chacko S, Sen I, Joseph E. Transseptal ascending aortic access and snare-assisted pull down of the delivery system to facilitate stent-graft passage in the aortic arch during TEVAR. J Endovasc Ther. 2013;20:223-30.
3. Dorros G, Dorros AM, Planton S, O'Hair D, Zayed M. Transseptal guidewire stabilization facilitates stent-graft deployment for persistent proximal ascending aortic dissection. J Endovasc Ther. 2000;7:506-12.

4. Kölbel T, Rostock T, Larena-Avellaneda A, Treede H, Franzen O, Debus ES An externalized transseptal guidewire technique to facilitate guidewire stabilization and stent-graft passage in the aortic arch. J Endovasc Ther. 2010; 17:744-9.

\title{
Minimally invasive robotically assisted surgical resection of left atrial endocardial papillary fibroelastomas
}

\author{
Alina Hua, MBBS, ${ }^{a}$ Richard J. Shemin, MD, ${ }^{\mathrm{b}}$ Jeffrey P. Gordon, MD, ${ }^{\mathrm{c}}$ and Eric H. Yang, MD, ${ }^{\mathrm{c}}$ London, \\ United Kingdom, and Los Angeles, Calif
}

\footnotetext{
From the Department of Medicine, ${ }^{\mathrm{a}}$ Imperial College London, London, United Kingdom; the Division of Cardiothoracic Surgery, ${ }^{\mathrm{b}}$ Department of Surgery, and the Division of Cardiology, ${ }^{\mathrm{c}}$ Department of Medicine, University of California at Los Angeles, Los Angeles, Calif.

Disclosures: Authors have nothing to disclose with regard to commercial support. Received for publication June 16, 2014; accepted for publication June 29, 2014; available ahead of print Aug 29, 2014.

Address for reprints: Eric H. Yang, MD, Division of Cardiology, Department of Medicine, University of California at Los Angeles, 100 Medical Plaza Suite 630, Los Angeles, CA 90095 (E-mail: Datsunian@gmail.com).

J Thorac Cardiovasc Surg 2014;148:3247-9

$0022-5223 / \$ 36.00$

Copyright (c) 2014 by The American Association for Thoracic Surgery

http://dx.doi.org/10.1016/j.jtcvs.2014.06.100
}

Cardiac papillary fibroelastoma (PFE) is the third most common type of primary cardiac tumor after myxoma and fibroma. It is known to be the most common valvular tumor and accounts for $5 \%$ of all tumors of the heart, although nonvalvular endocardial origins have been reported. ${ }^{1}$ Although cardiac PFE is considered a benign tumor, surgical excision may be warranted because of its propensity for causing embolic events and hemodynamic complications.

\section{CLINICAL SUMMARY}

An 89-year-old woman with a history of hypertension and breast cancer was seen after a witnessed syncopal 


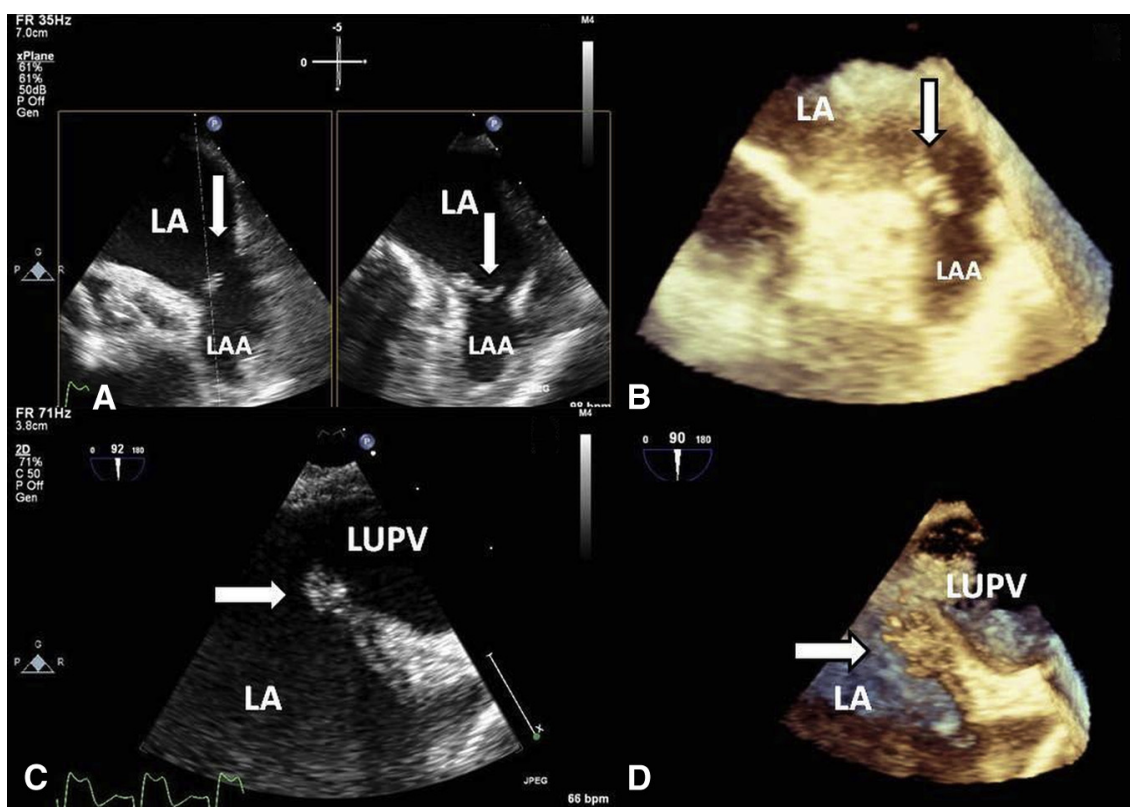

FIGURE 1. Transesophageal echocardiography, midesophageal view, showing the 2 cardiac masses. A, A 2-dimensional biplanar view of the first cardiac mass (arrow), measuring approximately $8 \times 7 \mathrm{~mm}$, which is seen at the ostium of the left atrial appendage and is adjacent to the atrioventricular circumflex groove. B, A 3-dimensional reconstruction of the mass (arrow). C, A 2-dimensional view of the second mass (arrow), measuring approximately $6 \times 9$ mm, at the tip of the left atrial ridge that divides the left atrial appendage and left upper pulmonary vein. D, A 3-dimensional reconstruction of the cardiac mass at the left atrial ridge. Both masses were mobile and appeared circular, pedunculated, and frondlike in appearance. $L A$, Left atrium; $L A A$, left atrial appendage; $L U P V$, left upper pulmonary vein.

episode. Magnetic resonance imaging of the brain revealed multiple infarcts within the posterior circulation. A transesophageal echocardiogram revealed 2 mobile echodensities, which were circular, pedunculated and frondlike in morphology (Figure 1). These masses were situated at the ostium of the left atrial appendage and at the tip of the left atrial ridge between the left upper pulmonary vein and left atrial appendage. The appearance of these masses led to a suspicion of PFEs, although other types of tumors and thrombus could not be excluded.

Invasive coronary angiography revealed mild, nonobstructive coronary disease. In the discussion of surgical strategies with cardiothoracic surgery, given that concurrent coronary artery bypass was not necessary, a minimally invasive robotic assisted approach with the da Vinci Surgical System (Intuitive Surgical Inc, Sunnyvale, Calif)

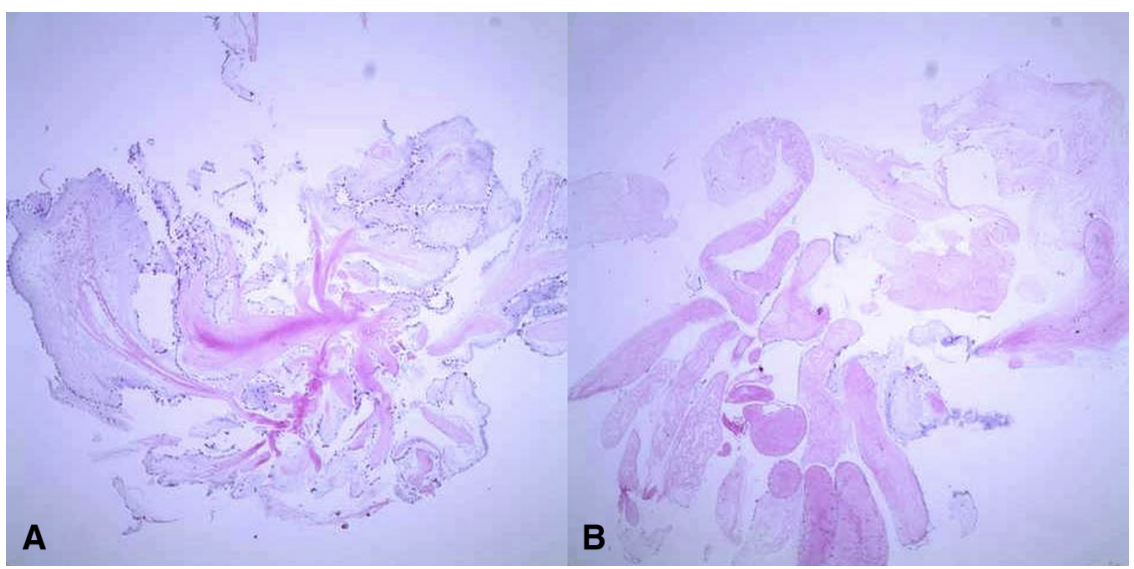

FIGURE 2. Hematoxylin and eosin stains of the surgically excised left atrial masses (original magnification $\times 4$ ). Both $($ A and B) show evidence of multiple frondlike structures with a collagenous core, consistent with papillary fibroelastoma. 
to resect the masses was proposed to avoid a sternotomy approach.

The patient was positioned for a robotic mitral valve approach. She was intubated with an endobronchial blocker to isolate the right lung. The superior vena cava was accessed with a $17 \mathrm{~F}$ cannula, and a cutdown was performed of the left femoral artery and vein. A small inframammary incision was made, and the chest was accessed through the intercostal space. Cardiopulmonary bypass was established and the right lung deflated; the pericardium was opened and tacked laterally. Antegrade cardioplegia cannula and venting needle were placed, and the aorta was crossclamped. Cold blood antegrade cardioplegia was initiated, arresting the heart.

With the da Vinci robotic system, robotic ports were placed in the chest and the left atrium was opened. Examination of the anterior left atrial cavity identified the two masses along the base of the left atrial appendage and along the inferior ridge of the left superior pulmonary vein, which visually appeared gelatinous, friable and myxomatous. The masses were excised and were extracted through a Lukens trap. The left atrial appendage was then closed. The atriotomy was then closed with GORE-TEX grafting (W. L. Gore \& Associates, Flagstaff, Ariz) and the aortic root was unclamped. The heart was then defibrillated to sinus rhythm.

The masses were confirmed to be endocardial PFEs on histopathologic investigation (Figure 2). The postoperative course was complicated by atrial fibrillation, which resolved with amiodarone. The patient was subsequently extubated, and she was discharged on postoperative day 11 in good condition. She currently continues to do well with minimal residual neurologic deficits and has had no recurrent neurologic events or evidence of cardiac tumor recurrence as of 1 year of follow-up.

\section{DISCUSSION}

A minimally invasive approach for atrial excision of masses may be an ideal strategy for patients who only require tumor resection because it eliminates the need for a sternotomy approach. Robotic minimally invasive mitral valve repair has been associated with decreased hospital stays, despite longer cardiopulmonary and aortic crossclamp times, and with faster recovery times. ${ }^{2}$ It has already been shown to be a potentially useful technique for cardiac myxoma excision. Gao and associates $^{3}$ were able to demonstrate that the da Vinci Surgical System allowed complete resection of cardiac myxomas in 19 consecutive patients with excellent clinical results, with no tumor recurrence at 18 months of follow-up.

The application of minimally invasive surgery to PFE excision has been limited to case reports. A few patients with PFE, including one with a mass in the right atrium, ${ }^{4}$ have been operated on through a minithoracotomy approach. The use of robotically assisted surgery to remove an aortic valve PFE has been reported..$^{5}$ Although there is potential in general for this technique in resecting cardiac tumors, clinical suspicion must be high for a benign cardiac tumor, and it is important to preserve the potential to change the surgical approach to an open sternotomy in the event that the tumor is found to be malignant with myocardial or pericardial involvement.

In summary, we present the case of a patient who was seen with a cerebrovascular embolic event caused by the presence and atypical location of left atrial PFEs, which were successfully removed by means of a robotic minimally invasive approach. This surgical technique can potentially be used for carefully selected patients with benign tumors who do not need surgical revascularization. In such cases, it has the potential to decrease bleeding, recovery time, and hospital stay.

We thank Annie Wu, MD, Michael Fishbein, MD, and Ali Sepahdari, MD, for their invaluable help in obtaining relevant data for the article.

\section{References}

1. Mariscalco G, Bruno VD, Borsani P, Dominici C, Sala A. Papillary fibroelastoma: insight to a primary cardiac valve tumor. J Card Surg. 2010;25:198-205.

2. Vernick W, Atluri P. Robotic and minimally invasive cardiac surgery. Anesthesiol Clin. 2013;31:299-320.

3. Gao C, Yang M, Wang G, Wang J, Xiao C, Wu Y, et al. Excision of atrial myxoma using robotic technology. J Thorac Cardiovasc Surg. 2010;139:1282-5.

4. Kim RW, Jeffery ME, Smith MJ, Wilensky RL, Woo EY, Woo YJ. Minimally invasive resection of papillary fibroelastoma in a high-risk patient. $J$ Cardiovasc Med (Hagerstown). 2007;8:639-41

5. Woo YJ, Grand TJ, Weiss SJ. Robotic resection of an aortic valve papillary fibroelastoma. Ann Thorac Surg. 2005;80:1100-2. 